\title{
Challenges with Deploying and Integrating Environmental Control and Life Support Functions in a Lunar Architecture with High Degrees of Mobility
}

\author{
Robert M. Bagdigian \\ NASA, Marshall Space Flight Center
}

\begin{abstract}
Visions of lunar outposts often depict a collection of fixed elements such as pressurized habitats, in and around which human inhabitants spend the large majority of their surface stay time. In such an outpost, an efficient deployment of environmental control and life support equipment can be achieved by centralizing certain functions within one or a minimum number of habitable elements and relying on the exchange of gases and liquids between elements via atmosphere ventilation and plumbed interfaces. However, a rigidly fixed outpost can constrain the degree to which the total lunar landscape can be explored. The capability to enable widespread access across the landscape makes a lunar architecture with a high degree of surface mobility attractive. Such mobility presents unique challenges to the efficient deployment of environmental control and life support functions in multiple elements that may for long periods of time be operated independently. This paper describes some of those anticipated challenges.
\end{abstract}

\section{INTRODUCTION}

NASA is continuing studies of various lunar architecture concepts and campaign strategies. By evaluating various options relative to a number of relevant figures of merit, these studies are helping not only to identify promising options which best meet the full range of exploration goals and objectives, but also uncover common themes and considerations that are likely to remain valid as lunar concepts and strategies continue to evolve. This paper will provide some insight into the architecture and campaign strategies that have been studied to date, to serve as a framework within which common themes and considerations applicable to lunar surface Environmental Control and Life Support System (ECLSS) development will be discussed.

\section{LUNAR SURFACE SYSTEMS}

NASA's exploration architecture concept envisions a combination of relatively short duration lunar sortie missions, with surface stay durations of up to 7 days, and longer surface missions of up to 180 days per crew rotation. Sortie missions will be conducted from crewed landers without dependence on any prepositioned surface assets, thereby enabling limited exploration of widely dispersed lunar locations of scientific interest. Additional exploration objectives can be met through the utilization of surface assets that can be incrementally deployed and integrated at a fixed location to create a more operationally-capable lunar outpost. Such a lunar outpost, shown notionally in Figure 1 , is expected to include habitats, logistics carriers, power generation and energy storage systems, surface mobility assets, science payloads, and in-situ resource utilization systems that enable the crewmembers to live, work, and explore the lunar surface in ways that would be unsupportable on lunar sortie missions. Habitable volumes will provide a pressurized, shirt-sleeved, controlled environment for the crew to live in and to conduct on-site scientific investigations.

One of the lasting lessons of the Apollo program is that surface mobility is key to improving the efficiency of humans on the lunar surface. To that end, NASA is currently developing surface mobility vehicles essential to meeting many lunar science objectives. One such vehicle that is being evaluated is a Lunar Electric Rover (LER), or equivalent, a prototype of which is shown in Figure 2. While smaller than a pressurized habitat, a 


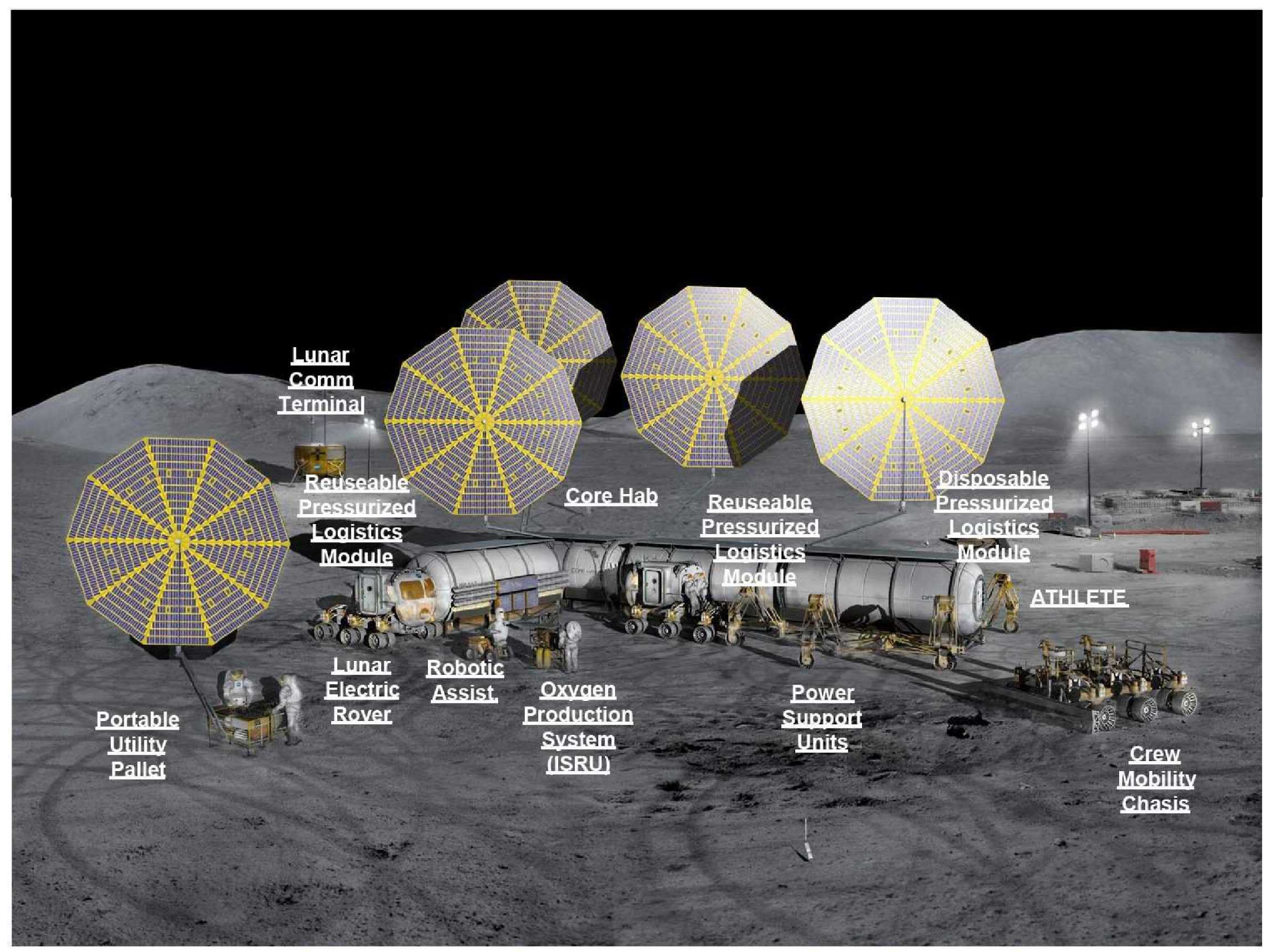

\section{Figure 1. Notional Elements of a Lunar Outpost}

pressurized rover can offer the capability to extend the reach of astronauts far beyond a fixed lunar outpost location while retaining access to laboratory equipment available in the outpost for increased scientific return. About the same size as the rovers used during Apollo Moon landings, a two-person rover could be equipped to handle three-day excursions with its own on-board resources. Longer excursions lasting up to 14 days could also be conducted with periodic resupply from transportable logistics carriers. Crew-time efficiency on excursions could be enhanced by configuring the rover with exterior-mounted spacesuits that the crew could don quickly through a rear-entry hatch and by controlling rover atmosphere conditions such that extended prebreathe periods are not required before the crew embarks on Extra-Vehicular (EVA). By reducing the operational overhead of exiting and re-entering the rover, multiple short duration EVAs can be performed within a single day, thereby increasing exploration flexibility to study widely dispersed lunar features as they are encountered.
The combination of short sortie missions conducted from lunar landers and longer missions centered at a fixed outpost serving as a hub for extended rover-based excursions offers many attractive exploration features. However, the development and efficient deployment of environmental control and life support equipment throughout a lunar infrastructure that includes both fixed and mobile assets must address some unique technical and operational challenges.



Figure 2. Prototype Lunar Electric Rover 


\section{LUNAR ECLSS CHALLENGES WITH MOBILITY}

GENERAL CONSIDERATIONS - General considerations related to the development of an integrated ECLSS for a lunar outpost were discussed previously (1). Given that an outpost will include some type of pressurized habitat(s) occupied by human crews for extended periods and that transportation costs will dictate that resupply rates be minimized to the lowest practical levels, the functions required of a lunar surface ECLSS can be defined with reasonable confidence. Those functions, along with the subsystems to which they may likely be assigned, are listed in Figure 3.
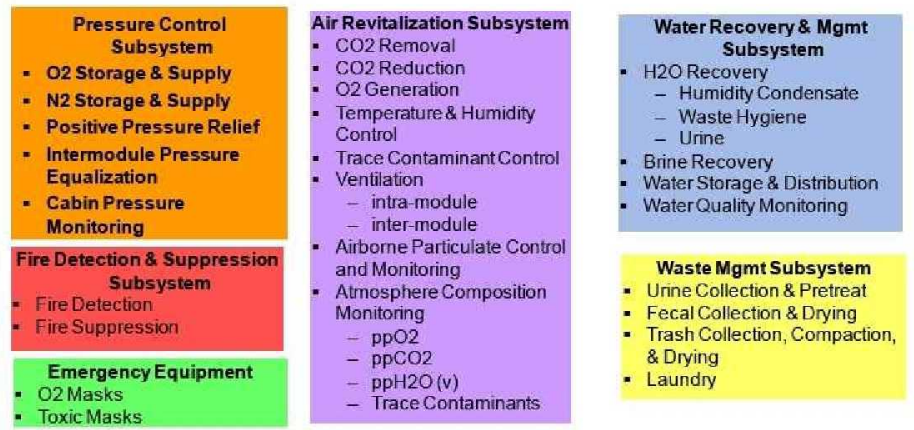

Figure 3. Lunar Surface ECLSS Functions (1)
In a complete lunar outpost made up of pressurized habitat(s), logistics modules, and rovers, it will be necessary to distribute the ECLSS equipment needed to perform the functions in Figure 3 among pressurized elements depicted in Figures 1 and 2 in a manner that supports outpost buildup and operations while avoiding unnecessary duplication of equipment. A simplified depiction of how ECLSS functions might be distributed among lunar outpost pressurized elements is shown in Figure 4

Centralizing much of an outpost ECLSS within a minimum number of pressurized habitat(s) can be an effective strategy in minimizing the amount of equipment that will be needed in volume- and resource-constrained pressurized rovers and in minimizing the tare weight of pressurized logistics modules. In such a strategy, intermodule ventilation can be used to maintain habitable conditions within attached pressurized elements. At the same time, extended crew occupancy of pressurized rovers operating remotely from the outpost dictates that a substantial amount of ECLSS functionality be provided independently in those rovers. And consideration must be given not just to the final end-state of a complete outpost.

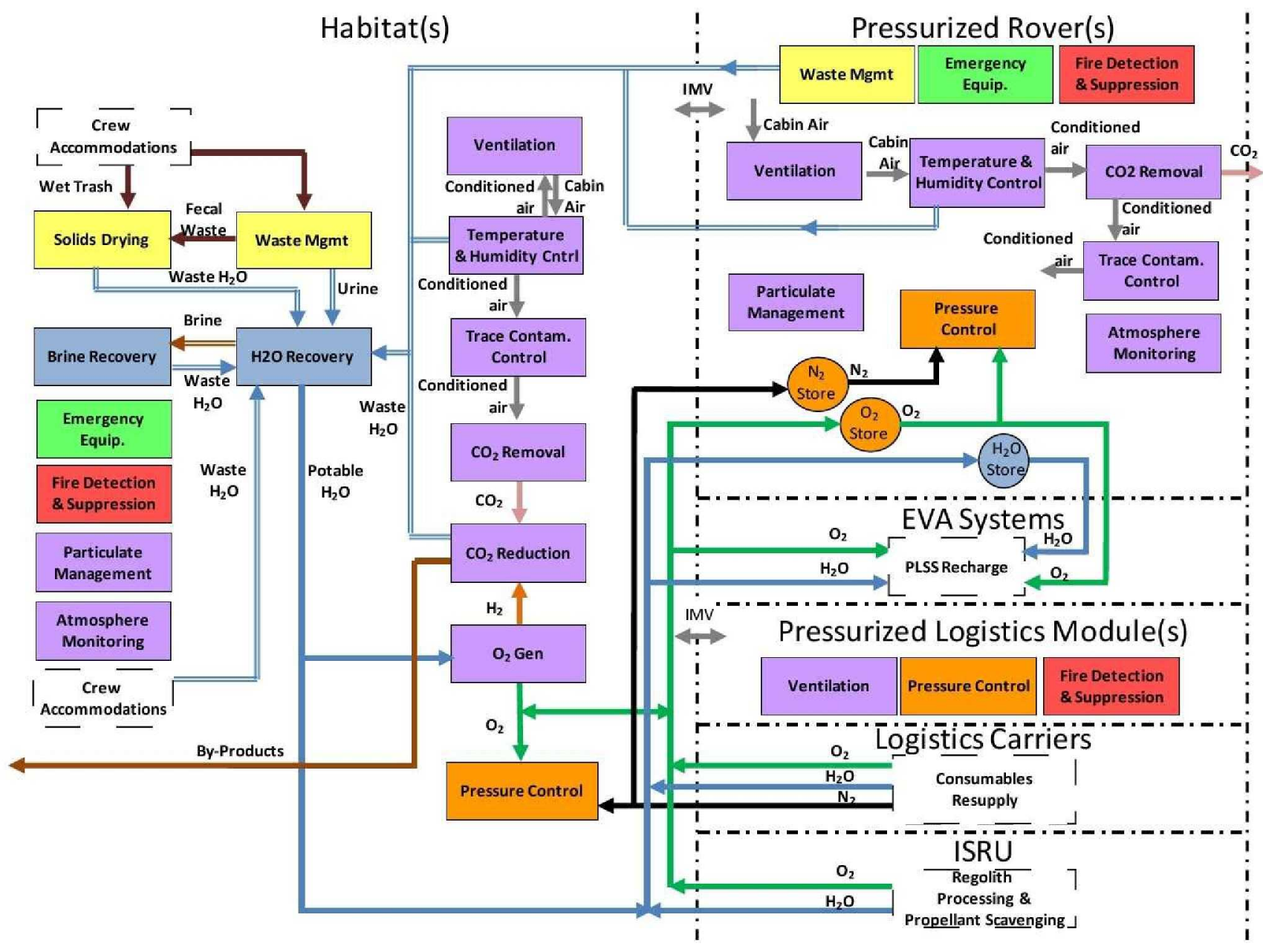

Figure 4. Distribution of ECLSS Functions Within a Representative Lunar Surface Architecture 
In any outpost architecture involving multiple pressurized elements that are incrementally deployed and occupied by crew, particular care will be needed in insuring that critical ECLSS functions are available with suitable fault tolerance throughout all phases of the build-up.

Oxygen and Water Loop Closure - It has long been recognized that provisioning extended duration human exploration missions with oxygen and water via "openloop" means (i.e., tanks) quickly becomes prohibitive as mission durations increase. A lunar surface system, occupied for months-long or continuous periods, can put a significant burden on transportation infrastructure to supply the oxygen and water needed to keep the crew alive, healthy, and productive while imposing substantial waste disposal demands. Loop closure, in which reusable oxygen and water are recovered from metabolic carbon dioxide and wastewater sources, provides a means for reducing this logistical burden. However, determining the appropriate degree of closure to pursue in development of a lunar surface ECLSS and how to effectively distribute and operate oxygen and water recovery systems located in various surface elements requires the balanced consideration of a number of factors.

A notional four-person daily metabolic mass balance is shown in Figure 5. The parameters represent a basic set of metabolic needs and wastes generated by an average crew of four persons in a typical day. Provided that the generated wastes are collected and accessible at the location(s) at which regenerative oxygen and water recovery systems are deployed, then it is possible to maximize the overall degree of loop closure attainable from a given set of oxygen and water recovery technologies. Furthermore, if the crew spends a high proportion of their lunar surface stay time within a lunar habitat, it is possible to localize the oxygen and water recovery systems in the habitat with minimal duplication across the lunar architecture. Such a condition exists on the International Space Station (ISS) where, except for rather infrequent EVAs in the immediate vicinity of the

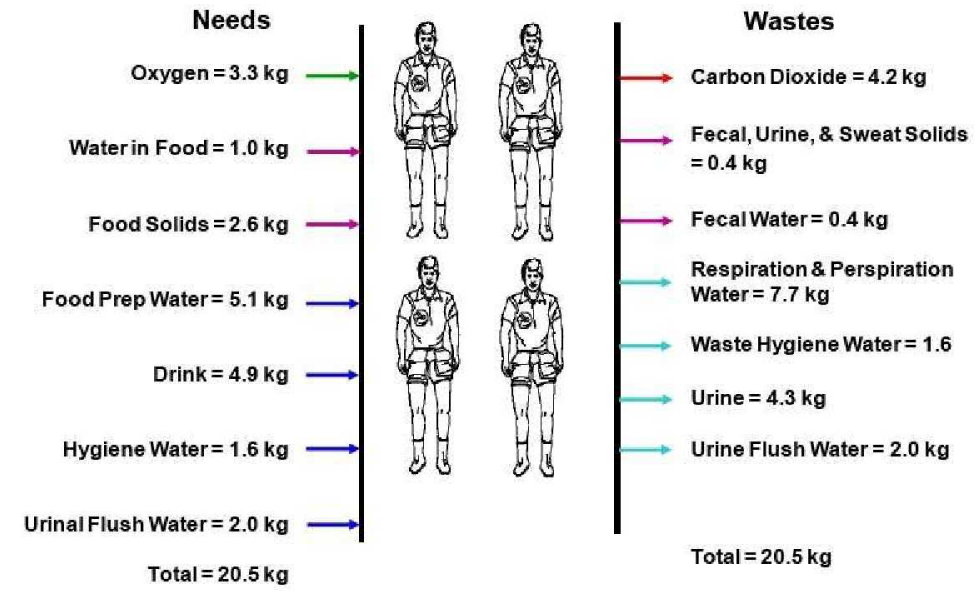

Figure 5. Notional Four-Person Daily Metabolic Mass Balance (1)
ISS modules, the crew spends virtually all of their onorbit time in an integrated assembly of habitable pressurized elements. That enables ISS oxygen and water recovery systems to be centralized in the U.S. Destiny Laboratory Module and the Russian Service Module. In general, oxygen and water are generated at rates approximating the daily needs from wastes that are collected and available for processing as they are produced.

A lunar outpost on the other hand will differ from the ISS in an important way. Most lunar exploration and science objectives will require the crew to spend a much larger percentage of their time outside the confines of the outpost habitats performing EVAs both in close proximity to the habitats as well as at relatively distant locations while on excursions in pressurized rovers. While a single 14-day excursion could be effectively conducted simply by carrying supplies of consumable gases and liquids and by discarding wastes along the way, such an open-loop approach would, over the course of repeated excursions over the operational life of an outpost, impose large logistics penalties on the lunar architecture as a whole. Yet the small confines within a rover and the need to keep the rover lightweight to maximize its translational energy efficiency conspire against burdening the rover itself with a full complement of water or oxygen recovery equipment. So the challenge becomes one of enabling efficient, frequent, and long rover excursions into an overall lunar architecture in a manner that is conducive to minimizing outpost logistics burdens through water and oxygen recovery.

Water vapor evolved from crew respiration and perspiration represents the largest fraction (over a third) of recoverable mass generated by crewmembers while inside a pressurized rover, totally more than $100 \mathrm{~kg}$ over a 14-day excursion. While water vapor could be removed from the cabin (along with carbon dioxide) through the operation of a swing-bed assembly, as is done with amine beds in the Orion crew cabin and the EVA Portable Life Support System (PLSS), analysis has shown that the addition of a condensing heat exchanger and regenerative water recuperator upstream of the rover's swing-bed assembly enables the collection of about $75 \%$ of the cabin water vapor that might otherwise be lost overboard. By adding provisions to store the humidity condensate, as well as the waste hygiene and urine that is also generated by the crew while in the rover, and returning those wastes to a centralized habitat where they can then be recycled for reuse. In this fashion, an appreciable logistical savings to the overall architecture can be achieved while keeping resource burdens on the rovers themselves low.

Carbon dioxide generated by crewmembers while in a rover also represents a potential recyclable resource. Current concepts for removing carbon dioxide from the rover cabin utilized amine-based pressure swing beds in order to maximize commonality with those that are currently baselined for use in the EVA PLSS. Having 
common beds in the rover and PLSS can enhance mission reliability and flexibility through operational redundancy and simplified spares management. Potential commonality paths that are being studied range from using identical copies of PLSS beds installed as integral parts of the rover's own vehicle ECLSS, using PLSS beds themselves to supplement the rover's beds in times of peak metabolic loading or as emergency backups, or relying on PLSS beds in place of rover beds entirely in order to reduce overall rover vehicle mass. Although attractive from a commonality perspective, a shortcoming of amines is that they are unsuited for thermal-swing desorption at elevated temperatures to capture, rather than vent, absorbed carbon dioxide for storage and later oxygen recovery back at a habitat. Other sorbent materials such as zeolites which are more amenable to thermal-swing desorption could enable the recovery of carbon dioxide from rover atmospheres. The attractiveness of such an approach could be enhanced if common zeolite beds can provide sufficient performance to meet EVA requirements and constraints while operating in a pressure-swing mode within a PLSS and meet IVA requirements while operating in a thermal-swing mode within a rover cabin. Equipment to compress and store desorbed carbon dioxide would represent rover resource burdens that would have to be carefully weighed. Furthermore, if the oxygen supplied for excursions is generated from water via electrolysis, it may prove worthwhile to store the by-product hydrogen at the habitat for later use in the reduction of carbon dioxide collected on LERs during excursions.

Preparing for Surface Excursions - The frequency, duration, and intervals between surface excursions will not be known with certainty for quite some time. And even after outpost assets are in place and operational, many factors will affect the excursion and EVA scheduling that will actually take place. It is therefore critical that outpost ECLSS architecture concepts be developed with consideration given to enabling maximum practical flexibility and not be rigidly optimized to an unrealistically narrow set of operational assumptions.

Current concepts conceive a number of pressurized rovers, each of which would be capable of sustaining two crewmembers for three days plus an additional two crewmembers in a 24-hour emergency return scenario. Longer excursions of up to 14 days total would be enabled through the use of portable utility pallets that could be carried by the rovers and dropped off at remote points away from the outpost cluster. In this manner; each pallet would have provisions for recharging rover oxygen and potable water supplies and accepting offloaded wastewater. In order to prepare for such extended excursions, wastewater must be offloaded and oxygen and potable water must be recharged onto the rovers and portable utility pallets after return from an excursion. Mission flexibility would dictate that the required preparation time between excursions be minimized, and therefore the equipment to be used for offloading wastewater and recharging oxygen and potable water be sized for highest practical throughputs. Power management, on the other hand, will likely favor lower throughput rates. To meet such opposing needs, it is likely that consumable oxygen and potable water for an upcoming excursion will need to be prepared in advance and stored at the outpost, perhaps while the crew and rover(s) are away on a previous excursion. Oxygen and water (both potable and wastewater) storage buffers will be needed at the outpost to accommodate such a capability.

Primary and secondary oxygen tanks within the PLSS are sized to meet EVA requirements when initially charged with gaseous oxygen (GOX) to 3000 psia. Therefore, oxygen supply equipment, both in fixed habitats at the outpost and in rovers from which EVAs will be conducted, will need to be able to deliver GOX at 3000 psia. A number of architectural approaches, each with differing technology needs, are being investigated to provide this capability. Water electrolysis will likely play a key role in closing the ECLSS oxygen loop in a lunar outpost. Water electrolysis is also a key intermediate step in the extraction of oxygen from lunar regolith (2) and in the regeneration of reactants in regenerative fuel cell energy storage systems (3). In each of these three applications, the capability to generate oxygen at pressures in excess of 3000 psia can offer attractive benefits in terms of overall system simplicity. However, electrochemical efficiency losses could limit electrolyzers to lower intermediate pressures which, in the case of ECLSS, would then dictate the need for supplemental compression to final storage pressures above 3000 psia. Extraction and compression of oxygen from ambient cabin atmospheres could also prove to be an attractive means of meeting the intermittent needs for high pressure oxygen. In all of these cases, removal (and recovery) of water moisture from the high pressure oxygen will be required in order to preclude condensation during storage. If liquid oxygen (LOX) supplies are available, the acquisition and compression of oxygen boil-off may be sufficient and potentially common with high pressure oxygen generation on the Altair lander. Equipment sizing in any of these cases will balance the goal of enabling quickturnaround excursions with architectural commonality and energy management.

Extended Periods When Habitats are Unoccupied Lunar campaign scenarios studied to date share a common attribute in that the capability to support continuous human presence requires the accumulation of a variety of assets and supplies that are collectively beyond the means of a single cargo lander to deliver. As a result, the early years of a campaign may be marked by prolonged periods of unoccupied dormancy during which emplaced elements will be required to be maintained in a safe, quiescent state without an attending crew. In fact, prior to launch, much of the equipment within a surface habitat can be expected to 
be in an unpowered, non-operational state while vehicle integration and launch preparations are completed. Once landed on the lunar surface, integrated habitats may remain mostly idle, with nothing other than a minimum set of "keep alive" functions operable until a visiting crew arrives, perhaps months later. The inevitability of prolonged periods of dormancy must be considered when developing ECLSS architectural concepts and while pursuing the technology development that will fill in the pieces of that architecture. This will be true regardless of whether a lunar architecture includes a high degree of mobility or not.

However, prolonged periods of untended presence won't end when continuous human presence on the lunar surface begins. Long duration excursions away from outpost assets will likely be routine. Immediately prior to these excursions, crew activities and equipment operation will likely be focused on preparing for the excursions, with recharging rover and utility pallet consumables a key task. As discussed previously, in order to facilitate minimum turnaround times between excursions, life support consumables will need to be prepared in advance. Once the crew departs the outpost, certain outpost ECLSS functions, like carbon dioxide and humidity removal, will inevitably transition towards idle mode as real-time metabolic loads subside. However, even though the crew themselves may be absent from outpost habitats, some ECLSS equipment will need to remain active and autonomously operated. Atmosphere pressures, composition, and temperatures will need to be continuously monitored and controlled as needed, perhaps at control points different from those maintained while the crew is present in order to reduce resource usage. Cabins will need to be continuously monitored for indications of fire and, if detected, alerts sent to the lunar crew and ground controllers while appropriate responses are implemented. And oxygen and potable water supplies for the next excursion will need to be replenished, in part from wastes off-loaded from rovers and utility pallets after the previous excursion. At the end of excursions, and before crewmembers transfer from rovers back into habitats, the habitat environments will need to be confirmed safe for entry.

Promoting High EVA Efficiency - The scientific return of human lunar exploration can be increased by minimizing the operational overhead associated with crewmembers preparing for and returning from EVAs. A novel means of facilitating rapid EVA egress and ingress is a suitport concept that is currently being explored by NASA (4). While offering many attractive benefits, one challenge that the suitport concept introduces is how to maintain suitable thermal and pressure control within the pressure garment while it is mounted to the suitport and unoccupied by a crewmember. Interfacing with the rover cabin air system is one approach that is being considered, provided that gas leakage losses are not prohibitive. Interfacing the pressure garment internal volume with a rover coolant loop has also been considered.

One of the bigger preparatory burdens for EVA are prolonged (depending on conditions) pre-breathe times that are required to remove dissolved nitrogen from crewmembers blood streams, protecting them from decompression sickness upon exposure to the lower ambient pressures within EVA pressure garments. To mitigate the risk of decompression sickness while avoiding prolonged pre-breathe periods, lunar pressurized atmospheres, including those in rovers, are expected to be controlled at or near total pressures of 8 psia. At 8 psia total pressure, the allowable span of acceptable oxygen partial pressure is tightly constrained on the low end to 2.48 psia (minimum allowable for continuous crew exposure after acclimation, per Reference 5) and on the high end to 2.72 (corresponding to $34 \%$ concentration material flammability limit, per Reference 6). This condition is depicted graphically in Figure 6 . With the pressurized volume of rovers currently estimated to be on the order of $11 \mathrm{~m} 3$, the ability to tightly control both total and oxygen partial pressures within the tight control bands necessary to maintain safe atmospheres under very dynamic conditions is likely to be a challenge.

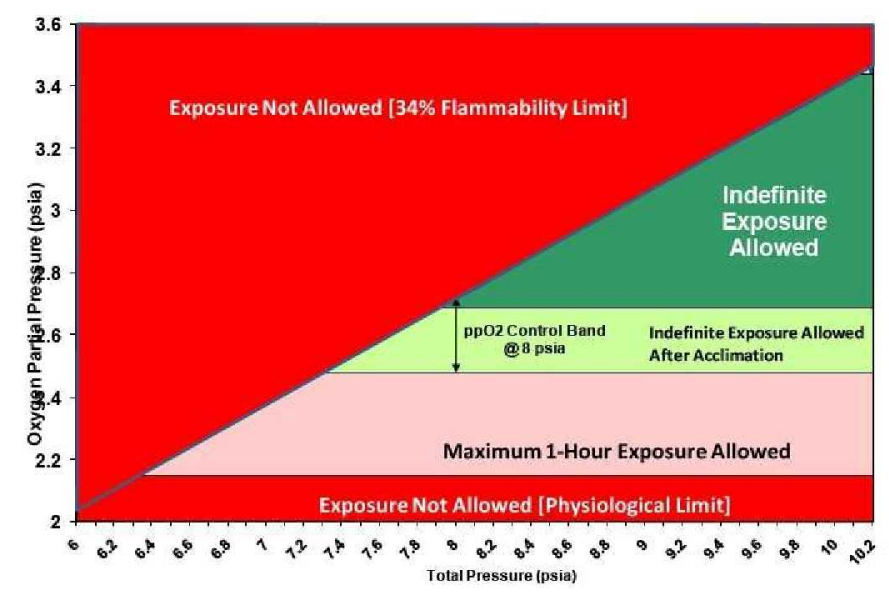

Figure 6. Allowable Oxygen Partial Pressures

\section{CONCLUSION}

Concepts for lunar outposts envision enhancing exploration capabilities and scientific return through enabling routine, wide ranging excursions across the lunar landscape. Such an outpost presents unique challenges to the effective and efficient deployment of ECLSS equipment among the various elements of the lunar outpost architecture. Some of those challenges have been presented in this paper.

\section{REFERENCES}

1. Bagdigian, Robert M., "Considerations Regarding the Development of Environmental Control and Life Support Systems for Lunar Surface Applications,", 
SAE 2008-01-2187, 38 ${ }^{\text {th }}$ International Conference on Environmental Systems, San Francisco, CA, July, 2008

2. Linne, Diane L, Joshua E. Freeh, and Andrew F. J. Abercromby, "Commonality of Electrolysis Subsystems for ISRU, Power, and Life Support for a Lunar Outpost", STAIF, 2008.

3. Bents, David A, Vincent J Scullin, Bei-Jiann Chang, Donald W. Johnson, Christopher P. Garcia, and lan J. Jakupca, "Hydrogen-Oxygen PEM Regenerative Fuel Cell Development at the NASA Glenn Research Center", NASA-TM-2005-214302

4. NASA Tech Brief, "Docking Fixture and Mechanism for a Protective Suit", ARC-14102, March, 2003

5. "Constellation Program Human-Systems Integration Requirements", CxP 70024, Rev. C, October, 2008.

6. "Constellation Architecture Requirements Document", CxP 7000, Rev C, December, 2008.

\section{CONTACT}

Robert M. Bagdigian

Constellation Lunar Surface Systems Project

Marshall Space Flight Center

Huntsville, AL 35812

robert.m.bagdigian@nasa.gov 